\title{
Numerical modelling of embankments and underground works
}

\author{
Ph. Mestat ${ }^{\mathrm{a}, *}$, E. Bourgeois ${ }^{\mathrm{a}}$, Y. Riou ${ }^{\mathrm{b}}$ \\ a Laboratoire Central des Ponts et Chaussées, 58 boulevard Lefebvre, Paris cedex 75732, France \\ b Laboratoire de Génie Civil de Nantes Saint Nazaire, Ecole Centrale de Nantes, 1 rue de la Nöe, BP 92 101, Nantes cedex 3 44321, France
}

\begin{abstract}
This paper deals with a bibliographic database dedicated to the comparison between FEM results and in situ measurements for geotechnical structures. This database, called MOMIS, has been developed by LCPC (the French Public Works Research Laboratory) for several years. To date, it comprises a total of 416 case studies, among which 133 deal with embankments on soft soils and 135 with underground works. The generation of MOMIS has relied on a technologic watch in the field of finite element modelling. The database can be used to highlight FE modelling principles (in order to provide a guide for good finite element practice) and to quantify deviations between results given by numerical models and values measured on actual geotechnical structures. It also reflects the evolution of the accuracy of numerical computations and trends in the simulation strategies.
\end{abstract}

Keywords: Modelling; Finite element method; Embankments; Tunnels; Measurements; Validation

\section{Introduction}

From a historical perspective, a wide array of studies have been conducted in order to compare numerical results with measurements on full-scale geotechnical structures and to validate computational models. The validation procedure must confirm the capacity of a model to resolve in a satisfactory manner a geotechnical problem under conditions resembling those of a project. Unfortunately, while this practice is the only one with any real pertinence, it remains complex as simultaneous testing has to be conducted on a software application, a theoretical model, the geotechnical tests for the determination of the parameter values and potential means of use. The computation hypotheses employed are of the utmost importance: they make it possible to run the tool and, in many instances, to compensate for a lack of data. Hypotheses must nonetheless be chosen with care; as such, modelling and engineering precepts are invoked to help ensure that these hypotheses remain representative. From these computation-measurement comparisons, it is necessary to highlight the modelling principles and to give information about a good practice.

\footnotetext{
${ }^{*}$ Corresponding author. Fax: +33-1-40-43-65-16.

E-mail addresses: philippe.mestat@1cpc.fr (P. Mestat), emmanuel. bourgeois@lcpc.fr (E. Bourgeois), yvon.riou@ec-nantes.fr (Y. Riou).
}

In order to study and to preserve the record of these validations, to draw lessons from these comparisons as well as to derive recommendations for geotechnical modelling (choice of model, assumptions and guidelines for controlling results) and to quantify the model error, the MOMIS database ("Structures Modelling and In Situ Measurements") has been developed.

Apart from two qualitative compilations, by Duncan [4] and Gens [5], and a qualitative study devoted to undergrounded facilities [12], no quantitative assessment has been published on the discrepancies between finite element results and in situ measurements. In light of this lack of references necessary for evaluating the capacity of numerical models to reproduce complex situations, the Geotechnical Structures Computations Unit of LCPC (Laboratoire Central des Ponts et Chaussées) has been conducting a technology watch mission to monitor these comparisons and estimate the resulting "model error" [9-11].

\section{MOMIS database}

The information extracted from bibliographical analysis have been combined into the MOMIS database. It comprises case studies originating from research studies conducted by the LPC network of laboratories 
(Public Works Regional Laboratories), from papers, conference papers and $\mathrm{PhD}$ theses. The data set extends back to 1972, with entries being evenly distributed over time. Nearly $75 \%$ of the references appear in conference proceedings, $20 \%$ stem from papers and the remaining $5 \%$ or thereabouts from reports or theses. The vast majority of modelling efforts recorded pertains to class $\mathrm{C}$ predictions (i.e., a posteriori prediction, following the classification by Lambe [6]). The references pertaining to class A predictions (i.e., before construction) are relatively infrequent.

At the end of 2002, the database MOMIS contains 416 case studies corresponding to the modelling of embankments on soft soils (84), embankment on improved soils (29), tunnels (135), sheet-piled retaining structures (66) and diaphragm walls (102) (both classes A and C predictions). These numbers seem high enough to make it possible to produce a statistical overview covering a 30 year period of finite element modelling (2D or 3D) and computation-measurement comparisons. Table 1 shows the breakdown of comparisons for 2D FEM results only.

For the same experimental case, several numerical studies may thereby be generated; such is the case for example when holding a blind prediction competition with several participants using different codes and constitutive laws. The aspects of modelling stored in the MOMIS database are: type of analysis (drained vs. undrained condition, consolidation, dynamic, cyclic); type of ground; construction technique employed, actual dimensions of the structure; constitutive laws for natural

Table 1

Number of comparisons with 2D FE analysis

\begin{tabular}{lr} 
Embankments (end of construction) & 39 \\
Maximum settlement on center line & 24 \\
Maximum lateral displacement in depth (toe of slope) & 16 \\
Maximum excess pore pressure on center line & \\
Embankments (long term) & 38 \\
Maximum settlement on center line & 26 \\
Maximum lateral displacement in depth (toe of slope) & \\
Embankments on improved soil (short term) & 35 \\
Maximum settlement on center line & 21 \\
Maximum lateral displacement in depth & 22 \\
Maximum excess pore pressure on center line & \\
Tunnels (end of construction) & 120 \\
Maximum surface settlement (transversal model) & 87 \\
Point of inflexion of settlement trough & 30 \\
Crown settlement & 32 \\
Maximum horizontal displacement & \\
Sheet-pile walls (end of construction) & 69 \\
Maximum horizontal displacement of the wall & 37 \\
Maximum settlement behind the wall & \\
Maximum bending moment in the wall & 24 \\
Diaphragm walls (end of construction) \\
Maximum horizontal displacement of the wall \\
Maximum settlement behind the wall \\
Maximum bending moment in the wall \\
\hline
\end{tabular}

soils and construction materials; values of computational parameters; computational model (dimensions, type of finite elements, mesh density, boundary conditions, loadings, time step, construction project phasing, interface laws, etc.).

An electronic version using the ACCESS database management application is being implemented in coordination with the Civil Engineering Laboratory of Nantes Saint Nazaire (Ecole Centrale de Nantes). Before the release of this computerized version, manual database operations with tables have enabled drawing some valuable lessons from nearly 30 years of modelling efforts on geotechnical structures. The displacements and other quantities were estimated from curves provided in the reference publications. Only a very small percentage of numerical values have been recovered directly from the papers.

\section{Modelling of embankments on soft soils}

\subsection{Description of the embankments included in the database}

Fig. 1 gives the number of case studies included in the base as a function of the height of the embankment. It shows that most embankments in the database have a height of 1.5-15 m. Given that embankments quite often exhibit a much greater length than width, their performance is typically studied using a transversal section in plane strain (see Fig. 2). Actual three-dimensional models are infrequent as they are not warranted.

When the fills are built on square-shaped bases, equivalent models can be derived using rotational symmetry provided that both the loadings and ground characteristics also respect these imposed conditions.

\subsection{Geometrical model}

The data contained within the MOMIS database serve to analyse the characteristics of meshes used in

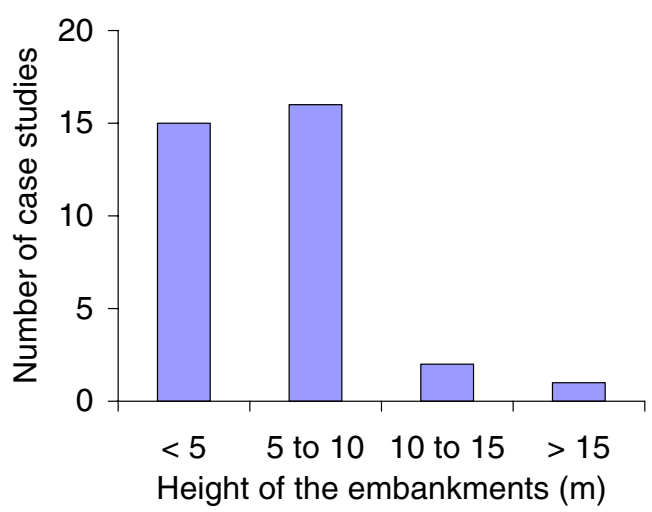

Fig. 1. Number of case studies vs. total height of the embankments. 
both vertical and horizontal directions for isolated structures built on soft soil (Fig. 2). Fig. 3 shows the relationship observed between embankment height $H$ and ground depth $h$, which represents the distance at which displacement boundary conditions have been imposed. The $h / H$ ratio varies between the extreme values of 1.4 and 15 , with an average equal to around 4 . Similarly, Fig. 4 describes the relationship between the

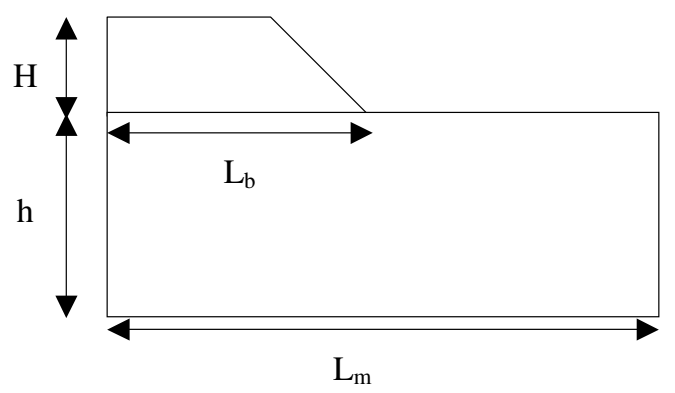

Fig. 2. Characteristic dimensions of a transverse section embankment model.

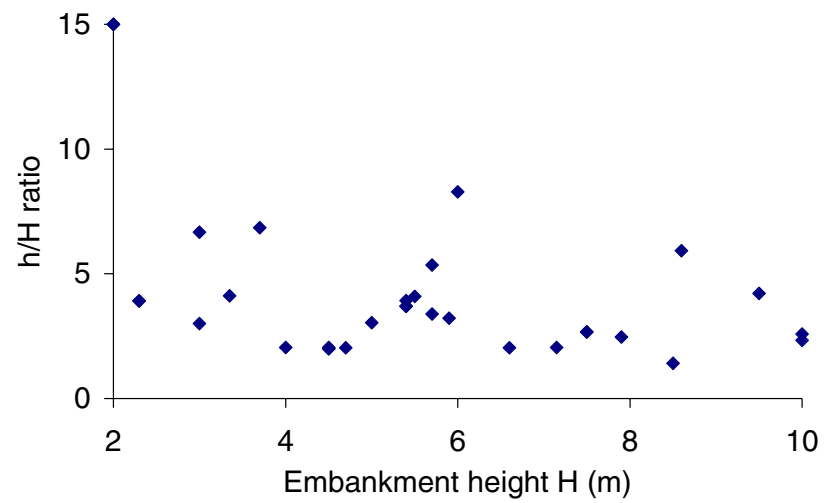

Fig. 3. $h / H$ Ratio as a function of embankment height (extracted from MOMIS database).

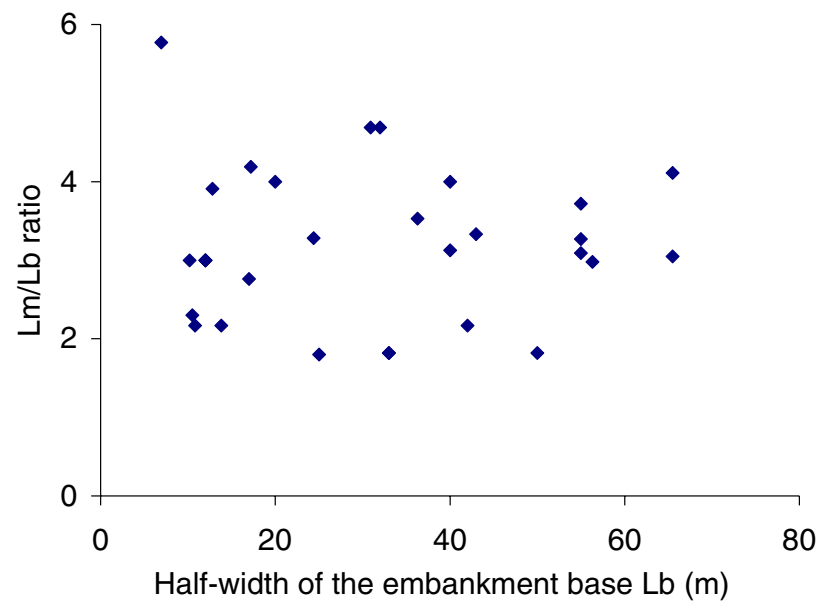

Fig. 4. $L_{\mathrm{m}} / L_{\mathrm{b}}$ ratio as a function of the half-width of the embankment base (extracted from MOMIS database). width of the embankment base $L_{\mathrm{b}}$ and the maximum model length $L_{\mathrm{m}}$, in order to represent the boundary conditions at infinity. The $L_{\mathrm{m}} / L_{\mathrm{b}}$ ratio lies between 1.8 and 5.8, with an average value of around 3 .

For those model set-ups which need not incorporate the presence of a substratum near the surface, obstacles or other interactions, this analysis serves to justify the minimum recommendations for earth structure models $[2,7,8]$

$h=4 H \quad$ and $\quad L_{\mathrm{m}}=3 L_{\mathrm{b}}$.

Enhanced computing power and capacity over the years has led to increasing the number of nodes taken into consideration in meshes, although this increase is not as large as could be expected. As computation speed continues to rise, more complex problems have been solved involving larger number of time steps, modelling of construction sequence and the complexity of the constitutive laws of soils. The number of nodes in recent models has thus remained limited to approximately 1500 for a two-dimensional transverse section embankment mesh (which is also symmetric with respect to the centre line). The vast majority of relevant analyses have been conducted in plane deformation. It is difficult to describe the mesh density, given that authors tend to offer little comment on the choices made for geometrical discretisation.

\subsection{Features of the analysis of embankments}

Certain experimental embankments were built to a point of reaching failure, while others were monitored over a long period of time. Computation-measurement comparisons were conducted either upon completion of the embankment or over a long period of time (corresponding to a measurement period of between 250 and 2200 days, depending on embankment height and soil compressibility properties).

For finite element numerical modelling, three theoretical approaches were performed: $20 \%$ of the references correspond to undrained conditions, $13 \%$ to drained conditions and $67 \%$ of analyses are coupled. Fig. 5 shows that there is a clear evolution towards the use of coupled analysis.

The majority of the embankments studied have been built on soft soils, and in some instances on very soft soils. The constitutive laws employed to describe the behavior of such soils are the following: linear and non-linear elasticity; elastoplasticity without strain hardening; elastoplasticity with strain hardening and elasto-viscoplasticity (Fig. 6). The most heavily used laws were developed from experimental and theoretical work conducted at the University of Cambridge. The modified Cam-Clay model was the most frequently applied among the other models $(35 \%$ of all references and $67 \%$ of all elastoplastic laws with strain hardening cited in the references). Over the past 10 years or so, the 


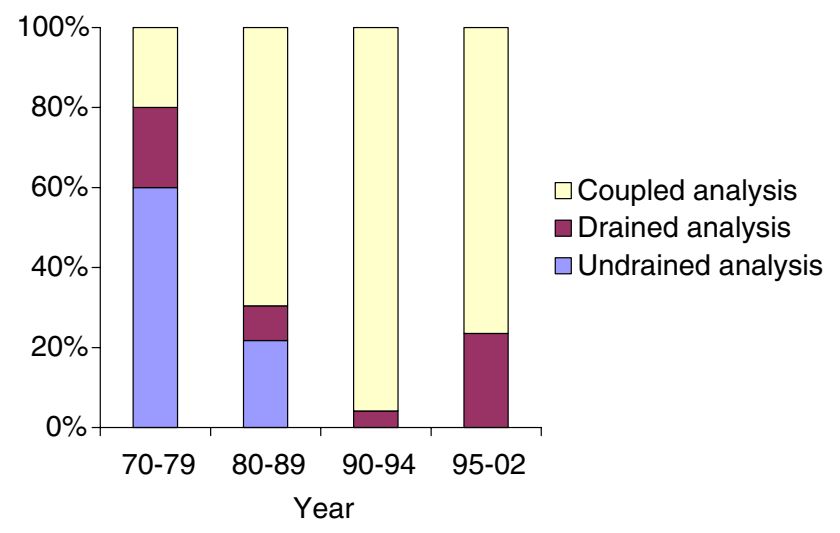

Fig. 5. Evolution of the type of analysis (with respect to the influence of pore pressure): drained, undrained or coupled analysis.

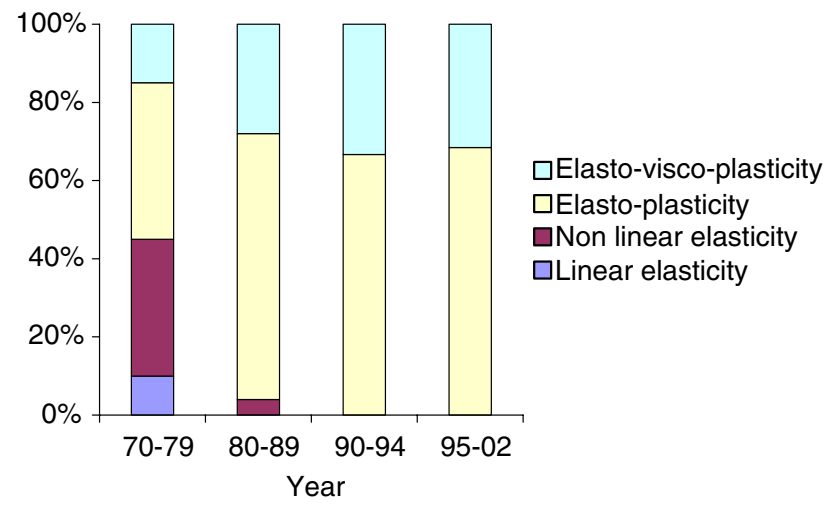

Fig. 6. Evolution of the type of constitutive model used in numerical models for soft soils over the past 30 years.

trend has favored use of elastoplastic models coupled with empirically based creep laws. Anisotropy is another phenomenon being incorporated by more recent models.

The embankment effect can be included within finite element models in two distinct ways:

- either by applying a pressure to the surface of the natural ground $(30 \%)$;

- or by representing the embankment by means of finite elements, gravity loading and a constitutive law $(70 \%)$. The mesh corresponding to the embankment may be activated all at once or according to a construction sequence in several successive layers. This approach is more frequently encountered in recent publications.

When the embankment is described by finite elements, the most widespread constitutive law is isotropic linear elasticity $(55 \%)$, followed by perfect elastoplasticity $(36 \%)$ and non-linear elasticity $(9 \%)$. The choice of such simple models stems from the limited information available on both the materials and their mode of implementation (effect of compaction, non-saturation, etc.).

\section{Computation-measurement comparison for embank- ments}

Comparisons between measured and computed values were made at the end of construction and over the long term. The studied variables are:

- the maximum vertical displacement on the surface along the embankment axis (Fig. 7(a) for short term and Fig. 7(b) for long term);

- the maximum excess pore pressure along the embankment axis (Fig. 8);

- the maximum horizontal displacement at the toe of the embankment (Fig. 9(a) for short term and Fig. 9(b) for long term).

For each variable analysed, the relative error was defined as the difference between the computed valued and the measured value, divided by the measured value. It may be useful to recall that the measured values are themselves subject to various errors inherent to the monitoring process and that it is therefore pointless to look for a perfect agreement between computations and measures.

Most class $\mathrm{C}$ predictions have led to relatively satisfactory results for vertical displacements on the surface along the embankment axis. Upon completion of
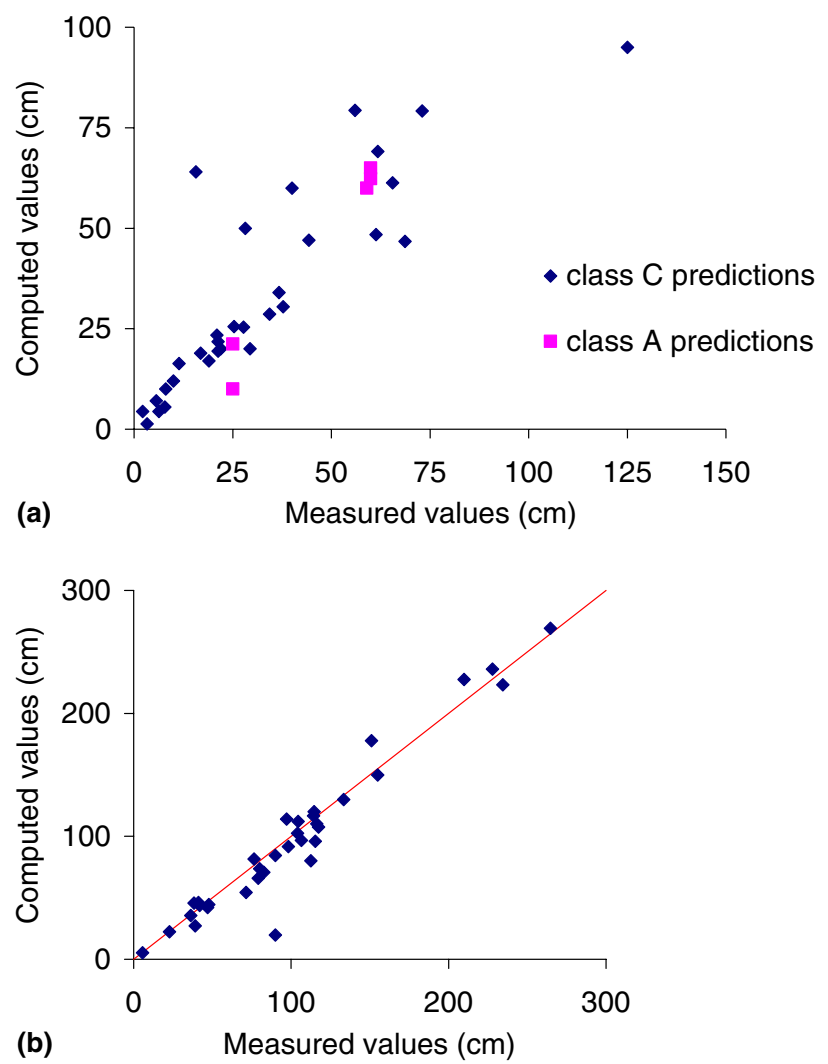

Fig. 7. Comparison between the computed settlement and measured settlement along the embankment axis: (a) at the end of construction and (b) over the long term. The straight line represents the bisector. 


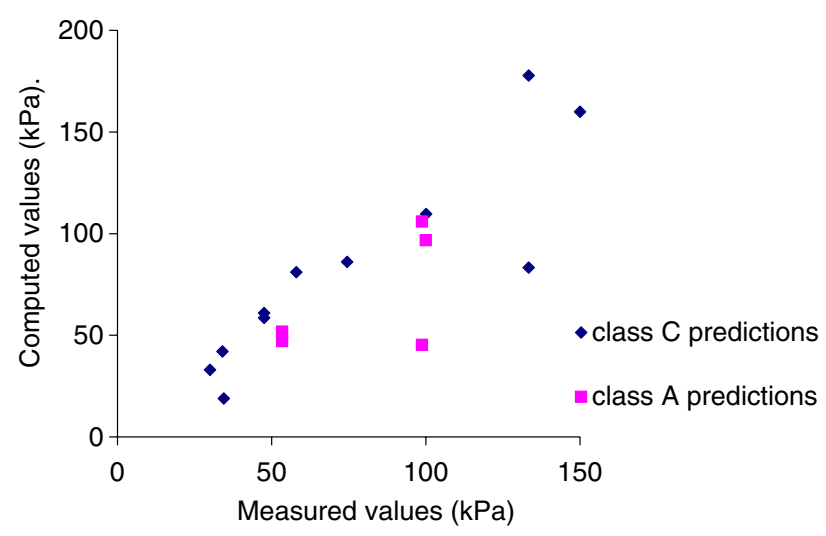

Fig. 8. Comparison in the maximum excess pore pressure between computed values and measured values along the embankment axis at the end of construction (extracted from MOMIS database).

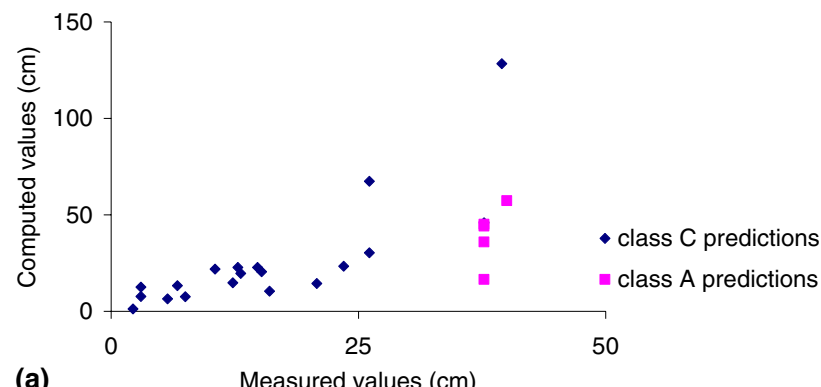

(a)

Measured values $(\mathrm{cm})$

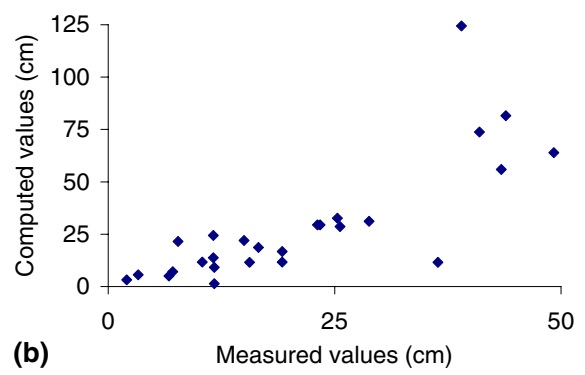

Fig. 9. Comparison in maximum lateral displacements between computed values and measured values at the toe of the embankment: (a) at the end of construction (extracted from MOMIS database) and (b) over the long term (extracted from MOMIS database). The straight line represents the bisector.

construction, the points corresponding to computation values and measured values are primarily located within the boundaries defined by the results from class A predictions (see Fig. 7(a)). For most of the computations (approx. 67\%), the relative error on surface settlement at the end of construction is less than 25\% (Fig. 7(a)).

Over the long term, it can be observed that both the computation and measurement points do not lie a great distance away from the bisector (Fig. 7(b)). The best modelling efforts are obtained with the help of elastoplastic laws with strain hardening, even if a few calibrations (whether admitted or not by their authors) have been performed. In certain cases, the use of elasto- viscoplasticity was necessary in order to assess the magnitude of observed movements. Ninety percent of the references display a relative error of less than $25 \%$ and the maximum error reaches $80 \%$ (Fig. 7(b)). Longterm settlement therefore is generally better described than end-of-construction settlement. The models incorporating elastoplasticity with strain hardening or elastoviscoplasticity tend to generate the smallest relative errors.

For the maximum excess pore pressures along the embankment axis, the estimated relative error is slightly higher (Fig. 8). Sixty-two percent of the predictions lead to an error of less than $25 \%$. The quality of predictions, focused on the global evolution in excess pore pressure, is also acceptable even though the uncertainties introduced in determining hydraulic parameters may at times be substantial. Drawing any kind of conclusion at this juncture would be difficult given the shortage in data available on pore pressures with respect to settlement measurements.

For the maximum lateral displacements at the toe of the embankment, the relative error at the end of construction is higher: on average, on the order of $60 \%$ with maximum errors rising to over $250 \%$ (Fig. 9(a)). Only $37 \%$ of the models yield a relative error of below $25 \%$. As regards the long-term values, this proportion falls to $31 \%$ (Fig. 9(b)). From a general standpoint, the models are not yet able to satisfactorily describe the lateral displacements and often overestimate them.

\section{Modelling of underground works}

\subsection{Description of the underground works included in the database}

Despite the fact that underground structures, once built, are linear structures, the displacement and stress fields are heavily three-dimensional in the vicinity of the tunnel face during construction. Strictly speaking, construction simulation thus requires a three-dimensional model, which often leads to complex modelling and high computation times. In order to reduce the computational cost, several approximate methods have been proposed for simulating construction effects using a twodimensional model. All of these methods consist of studying the tunnel within a full (or half) cross-section (Fig. 10): the three-dimensional effects are taken into account by means of imposed loadings or displacements at the intrados. Eighty-two percent of the references contained in the MOMIS database pertain to plane strain modelling applications. Nonetheless, three-dimensional models have indeed been included and are becoming increasingly commonplace with the advent of more powerful computers (16\% of all MOMIS references). These models encompass fine-tuned analyses of 


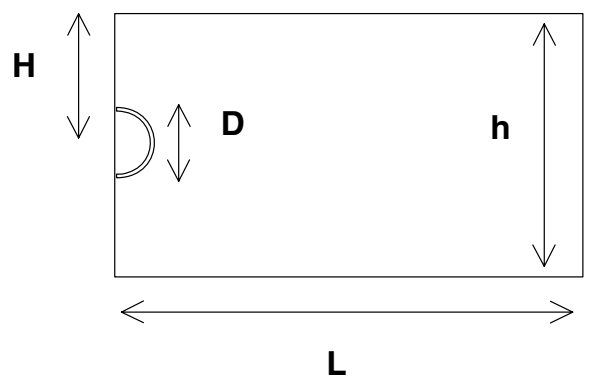

Fig. 10. Characteristic dimensions of a transverse section tunnel model.

tunnel face stability, gallery intersections, and underground cavities displaying complex geometries (factories, quarries, underground train stations). The other models describe a longitudinal section in plane strain or a meridian plane in rotational symmetry for cavities.

Approximately $20 \%$ of the case studies entail models closely adapted to the New Austrian Tunnelling Method ("NATM") simulation and $52 \%$ involve the shield tunnelling technique.

\subsection{Geometrical model}

We denote by $D$ the tunnel diameter (or the largest tunnel dimension in the case of non-circular tunnels), by $H$ the tunnel axis depth with respect to the natural ground surface, and by $h$ the depth (with respect to natural ground) at which displacement boundary conditions get imposed. Data extracted from the database show that the $h / H$ ratio varies between 1.2 and 5.3, with an average value of about 2.5. Similarly, if we denote by $L$ the distance at which lateral limits of the mesh (and therefore boundary conditions) are placed, the $L / D$ ratio lies in the range from 2 to 17.7, with an average value of around 5. This analysis justifies the minimum recommendations advised for underground structure models $[1,3,8]: h=2.5 H$, and $L=5 D$.

Even though the number of three-dimensional models is still too small to be representative, it should be pointed out that the equivalent ratios are of the same order of magnitude. Accordingly, the ratio of the threedimensional mesh height to depth $H$ stands at an average value of 2 while the tunnel length-to-diameter ratio comes to around 6 .

This ratio is probably too small to get a correct estimation of the final settlement above the tunnel axis and of the variations of the settlement with the distance to the tunnel face. In other words, it seems that threedimensional tunnel analyses tend to limit the possibilities of numerical simulations, in terms of number of nodes and of computation times needed to achieve the simulation of a significant length of tunnel.

Increasing computing power has resulted in an increase in the number of nodes introduced into meshes

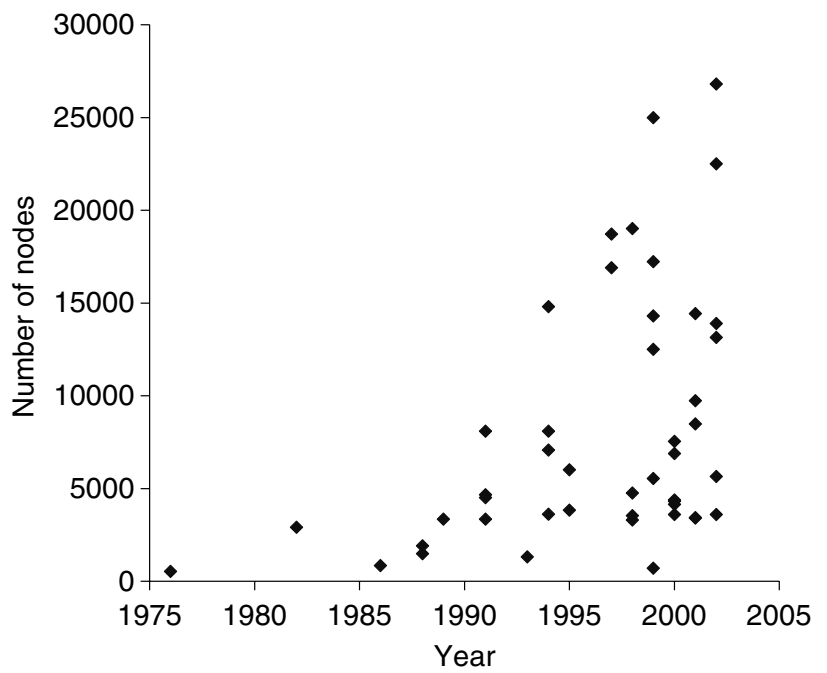

Fig. 11. Trend in the number of nodes used in $3 \mathrm{D}$ modelling of underground structures.

over time (see Fig. 11). This trend however is not necessarily as sharp as may have been expected. While the rise in computation speed is undeniable, it has not exclusively gone towards extending the level of mesh refinement; a considerable share of this gain in computation power has allowed boosting the number of iterations and load increments as well as adding complexity to non-linear soil constitutive laws and to construction sequence simulations. The number of nodes for recent models has, therefore, remained limited to an average of approximately 2500 for $2 \mathrm{D}$ cross-sectional meshes of underground structures (symmetrical about their axis) and 10,000 for $3 \mathrm{D}$ models.

As for embankments, three types of analyses were carried out: under undrained conditions, under drained conditions and consolidation (Fig. 12). The constitutive models used for describing soil behavior (whether excavated or not) encompass the following types of laws: linear and non-linear elasticity (these tend to comprise the oldest references); elastoplasticity without strain hardening; elastoplasticity with strain hardening and

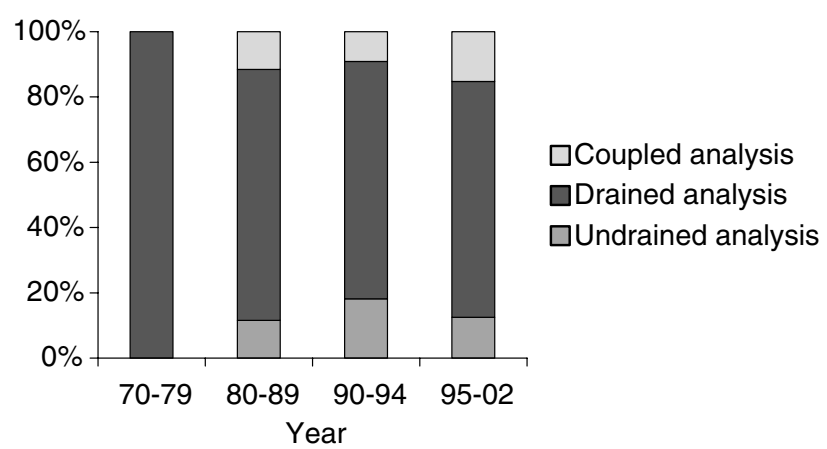

Fig. 12. Evolution of the type of tunnel analysis (with respect to the influence of pore pressure): drained, undrained or coupled analysis. 


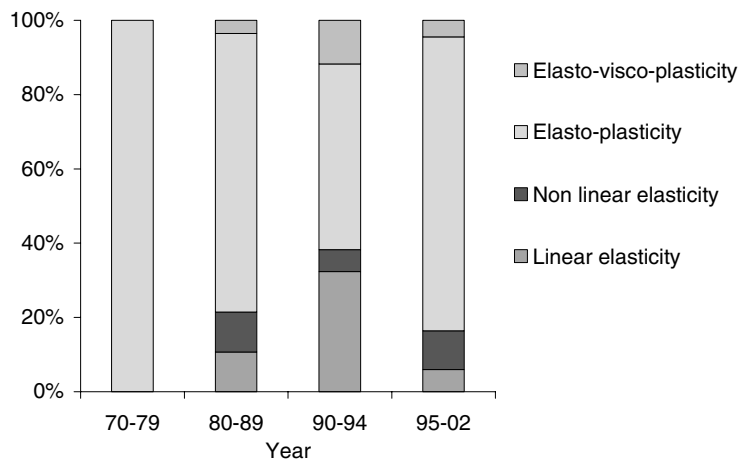

Fig. 13. Evolution of the type of constitutive model used in numerical models of tunnels over the past 30 years.

elasto-viscoplasticity (Fig. 13). Generally speaking, the most widespread constitutive law is the Mohr-Coulomb perfect elastoplasticity model with isotropic linear elasticity. Among the elastoplastic laws with strain hardening, the Cam-Clay model remains the most widely used. The complexity of the loads applied to tunnel models, along with the excavation simulations, have given rise to a simplification of the analyses and behavior under consideration. In addition, consolidation analyses prove to be somewhat rare for underground structures, whereas they have become essential to model embankments on soft soils.

The lining of a tunnel may be represented in the finite element model in one of the three ways: by applying pressure at the intrados; by imposing the displacements at the excavated zone boundaries (zero or nonzero displacements) and by representing the lining through combining finite elements (beam, shell or volume elements), a gravity load and a constitutive law. The corresponding mesh may then be activated either all at once or in accordance with a step-by-step phasing of the works. The last case is most frequently encountered among recent models. Truss or beam elements can also be considered for describing certain types of supports (bolts, anchorages, etc.). When the lining is described by finite elements, the most common constitutive law employed is that of isotropic linear elasticity.

The excavation may be simulated by adopting one of the two following methods:

- gradual softening, which consists of simulating the progress of the tunnel face in the soil by virtue of a gradual decrease in stiffness of the soil located within the excavation zone. Young's modulus of the corresponding elements is then reduced by a softening coefficient, whose value depends on the tunnel face position at a particular moment;

- forces due to confining-stress removal, which represent (via a wall pressure distribution) the ground decompression caused by progression of the tunnel face in the soil. This approach has proved to be prevalent.

\section{Computation-measurement comparison for tunnels}

The comparisons presented pertain to two-dimensional models and measurements carried out on crosssections. They focus on: maximum surface settlement along the tunnel axis, the location of the inflection point and the width of the settlement trough, and vertical and horizontal movements including crown settlement. The comparisons involving measurements of stresses, forces or moments within the lining are not presently available in adequate numbers to provide any meaningful insight.

Moreover, it should be noted that all comparisons presented do not take into account either the soil characteristics (cohesive or noncohesive), the construction process (NATM, divided sections, etc.), or the type of support and lining. All plane strain computation results have been represented in global terms. More refined analyses still need to be performed in order to determine whether the modelling approach is capable of better simulating one particular technique over another, since doing so requires rather extensive data. Once MOMIS has reached the point of containing sufficient relevant data, this set of more refined analyses will be undertaken.

At the soil surface, tunnel excavation-induced settlements are distributed transversally according to a roughly normal bell-shaped law [13]. This distribution depends upon the following two parameters:

- the maximum settlement on the tunnel axis $s_{\max }$,

- the distance $i$ between the tunnel axis and the inflection point of the distribution curve:

$s(x)=s_{\max } \exp \left(-\frac{x^{2}}{2 i^{2}}\right)$,

where $x$ is the distance to the tunnel axis.

For the maximum surface settlements $s_{\max }$ obtained at the end of construction, the points corresponding to computations and measurements are not too far from the bisector, except for a few of them (see Fig. 14). A very large majority of the points lie within the boundaries given by the two class A predictions. More precisely, for approximately $60 \%$ of the modelling set-ups, the relative error on maximum surface settlements is less than $25 \%$. The description of maximum settlement using simple elastoplastic laws is therefore satisfactory, provided the right model parameter values are available. Eight-two percent of the models identified in the database generate relative errors of less than $50 \%$. It is worth noting that this relatively good performance of numerical codes is interesting, since the a priori estimation of the maximum surface settlement by empirical or semianalytical methods is not as straightforward and reliable as the estimation of the other parameter of the settlement trough (parameter $i$ ).

In practice for the analysis presented hereafter, values of $i$ have been obtained on both the theoretical and 


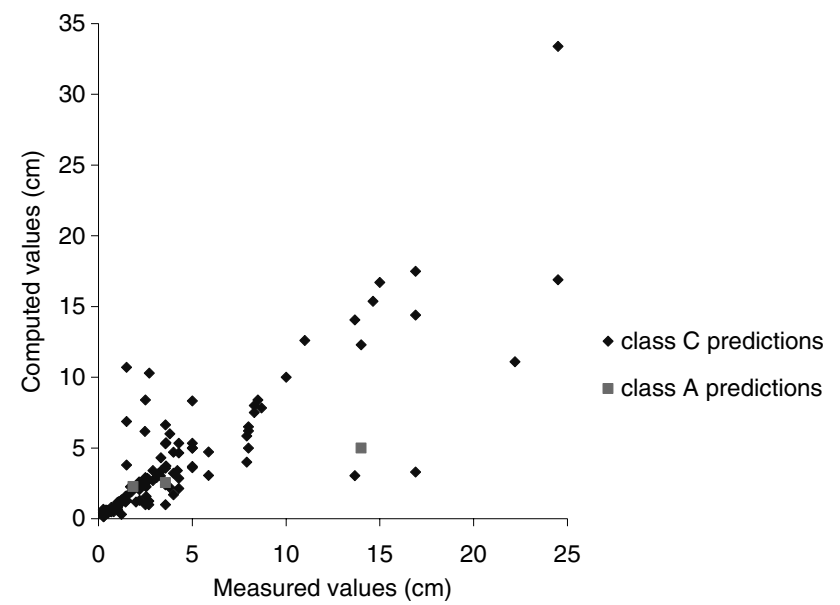

Fig. 14. Comparison of computed vs. measured maximum surface settlements (extracted from MOMIS database). (The squares represent class A predictions.)

experimental curves by estimating the distance to the tunnel axis for which the settlement corresponds to $60 \%$ of the maximum settlement (i.e., the settlement on the axis). This simplified approach has enabled a quick estimation of the parameter $i$ for those references where a settlement profile has been presented.

For $54 \%$ of the models, the relative error in inflection point position is less than $25 \%$. In general, the extent of settlement troughs has been quite well represented (see Fig. 15). Nonetheless, this figure does not reflect the models in their entirety since a good number of references provide measurements that do not enable deriving accurate estimates of trough width. It can also be noted that the use of models with strain hardening significantly improves trough simulation, as opposed to results obtained from an ideal elastoplastic model.

The measurements of both crown settlements and horizontal displacements are less commonly encountered than measurements of surface movements. A sufficient number of data points however are available.

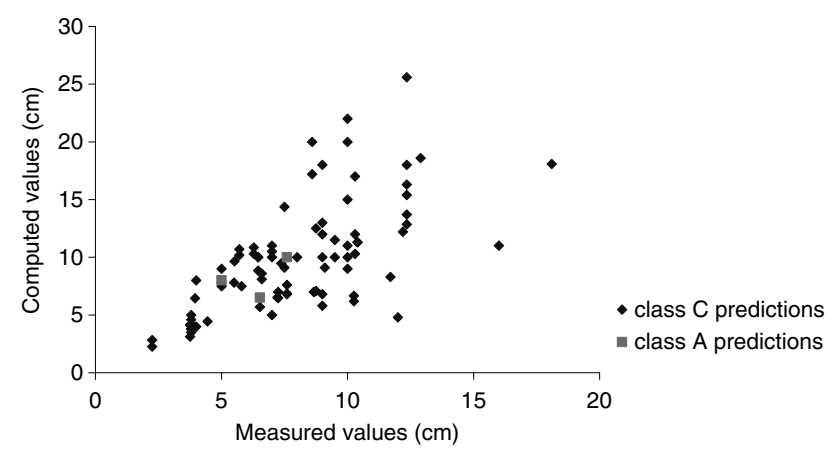

Fig. 15. Comparison of computed vs. measured $x$-axis coordinates of settlement trough inflection points (extracted from MOMIS database). (The squares represent class A predictions.)

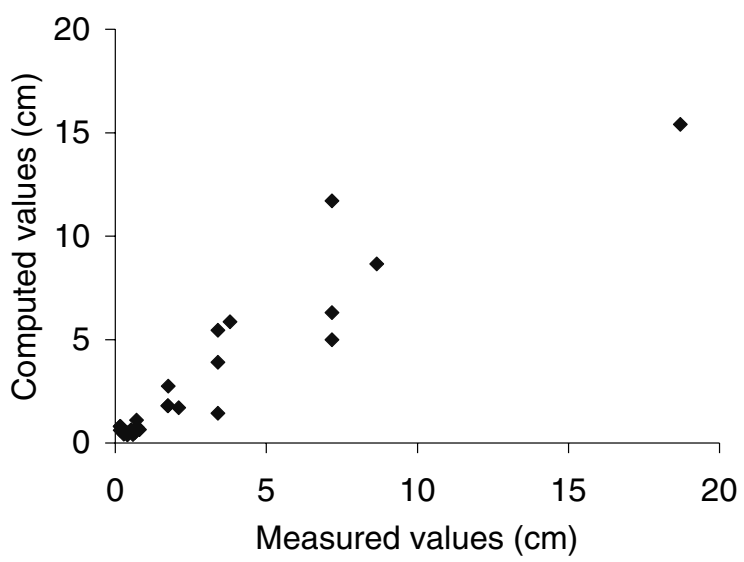

Fig. 16. Comparison of computed vs. measured wedged settlements (extracted from MOMIS database).

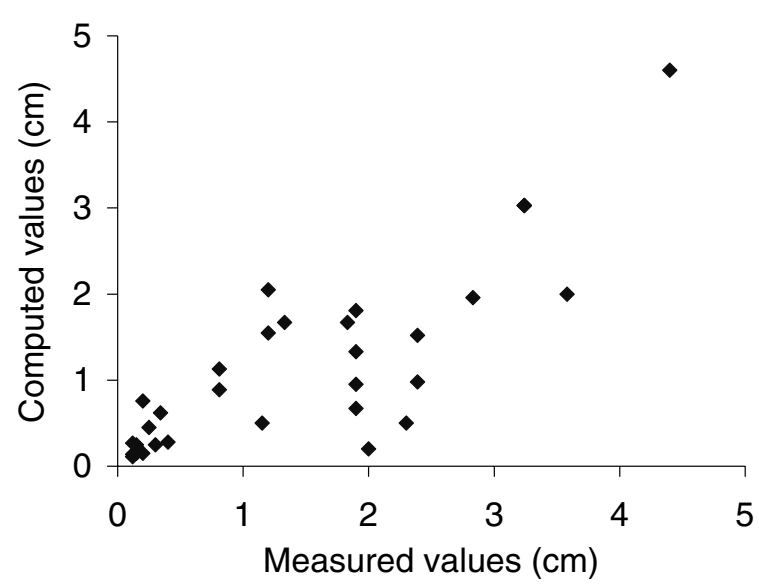

Fig. 17. Comparison of computed vs. measured maximum horizontal displacements (extracted from MOMIS database).

Maximum horizontal displacements were recorded on the inclinometer measurements closest to the tunnel axis. For crown settlements, approximately $56 \%$ of the models yield a relative error of less than $25 \%$ (see Fig. 16), whereas for the maximum horizontal displacements, this percentage falls to $43 \%$. Fig. 17 shows both the dispersion in results and the difficulty for models to satisfactorily describe horizontal movements. This theoretical difficulty is nothing new however; it was already observed during the study of models for embankments on soft soils.

\section{Towards the definition of an estimator of model error}

Developing a quantitative estimate of the global accuracy of the numerical simulations is a very complex problem. An estimate of the global validity of a numerical simulation method must include various aspects of the computed solutions, with various levels of 
reliability. This leads to the idea that the model error accrued during the computations (seen as the "sum" of the errors related to the software, its use, the approximated computation method, the approach employed to obtain the computational parameters and the modelling of the geotechnical work and its environment) may be quantified on the basis of comparisons between in situ measurements and computed results. In deriving this cumulative error, class A predictions are obviously ascribed higher priority. Unfortunately, they tend to be rather rare and it would be desirable for their number to expand over the coming years.

The previous analyses have considered relative errors with respect to the variables in an independent manner. From the idea that an effective model must be able to simultaneously predict all of the key aspects in the response of a geotechnical structure to the loadings imposed upon it, we may define a "cumulative error" which is equal to the sum of the absolute values of relative errors on several variables (for example, surface settlements and horizontal displacements, pore pressures). As a means to estimate this cumulative error, the entire series of references included in the MOMIS database were considered.

For embankments on soft soils, $70 \%$ of the models analysed reveal a cumulative error of above $100 \%$ at the end of construction. For comparison of the long term behavior, $83 \%$ of the models provide a cumulative error of less than $100 \%$ and just $65 \%$ show a cumulative error of below $50 \%$.

For tunnels, model error is estimated by means of summing the relative error in surface settlement on the tunnel axis with the maximum error accrued on either the crown settlement or the maximum horizontal displacement. For a computation-measurement comparison at the end of construction of tunnels, $14 \%$ of the models analysed provide a cumulative error of less than $25 \%$ and just $36 \%$ show a cumulative error of below $50 \%$. In contrast to this, $18 \%$ of the predictions reveal a cumulative error of over $100 \%$. The magnitude of the cumulative error stems from errors observed when simulating underground movements, especially around the tunnels.

\section{Conclusions}

A technology watch has been conducted over the past several years with respect to comparing finite element model results with measurements taken on actual fullscale structures. A wide array of papers, conference papers and research reports have been collated. The objectives are to preserve the record of these models and their comparisons with in situ measurements, to justify several state of the art rules in the practice of geotechnical modelling, to provide orders of magnitude for computation results, and to quantify the modelling error.

The information extracted from the bibliographical analysis has been combined into a database called MOMIS. Collation of the references has served to identify a few general modelling rules (e.g., for the mesh dimensions) along with some important conclusions, such as

- the elastoplastic laws without strain hardening remains the most widespread in representing the behavior of soil in retaining structure problems;

- for the modelling of embankments, the elastoplastic laws with strain hardening have now become the most widespread in representing the behavior of soft soils. The "cumulative model error" lies on average in the $75 \%$ range at the end of construction and around $60 \%$ over the long term. These high percentages are often due to the poor simulation of lateral displacements;

- for the modelling of tunnels, the cumulative model error lies on average in the $70 \%$ range at the end of construction. This high percentage is often due to the poor simulation of horizontal movements around the tunnels.

The disparity observed on computation-measurement curves is considered to be due to a combination of finite element method approximations, soil heterogeneity, the approach used to incorporate three-dimensional effects and the complexity of underground construction techniques. The level of dispersion is higher for horizontal displacements, for which the quality of model simulation falls short of being satisfactory.

The MOMIS database is just in the early stages of its development. Other aspects concerning embankments may soon be open to analysis, such as the determination of computational parameters (laboratory or in situ testing, correction of laboratory-derived values) and more in-depth soil-related studies. Other prospective applications for MOMIS include the introduction of computation-measurement comparisons for foundations and reinforced soils.

It is essential to continue conducting these computation-measurement comparisons along with additional full-scale experiments. The scientific community needs to ascertain the degree of realism in the models it is proposing in order both to adjust/refine them and to define modelling methodologies specific to each type of structure.

\section{References}

[1] Clough GW, Leca E. Modélisation et méthode de dimensionnement: utilisation de la méthode des éléments finis pour l'analyse des tunnels en terrain meuble. Colloque International Tunnels et Micro-tunnels en terrain meuble, Paris, 1989, Presses de l'ENPC. 
[2] Desai CS, Christian JT. Numerical methods in geotechnical engineering. New York: McGraw-Hill; 1977. 784 p..

[3] Duddeck H. Application of numerical analyses for tunnelling. Int J Numer Anal Methods Geomech 1991;15:223-39.

[4] Duncan JM. The role of advanced constitutive relations in practical applications. In: 14th International Conference on Soil Mechanics and Foundation Engineering. vol. 5. New Delhi, India: Oxford \& IBH Publishing Company; 1994. p. 31-48.

[5] Gens A. General report: prediction, performance and design. In: Shibuya $\mathrm{S}$ et al., editors. Pre-failure deformation of materials, vol. 2. Balkema; 1995. p. 1233-56.

[6] Lambe TW. Prediction in soil engineering. Géotechnique 1973;23(2):149-202.

[7] Lépidas I, Magnan J-P. Fluage et consolidation des sols argileux: modélisation numérique. Rapport de recherche LPC, 1990, no. 157, LCPC, Paris, $170 \mathrm{p}$.

[8] Mestat Ph, Prat Michel, Bisch Philippe, Millard Alain, PijaudierCabot G. Ouvrages en interactions. Paris: Editions Hermès Sciences; 1999. 944 p.
[9] Mestat $\mathrm{Ph}$. An overview on 25 years of numerical modelling of test embankments and tunnels. In: Desai CS et al., editors. Computer methods and advances in geomechanics. Balkema; 2001. p. 15216.

[10] Mestat Ph. MOMIS: une base de données sur la modélisation numérique des remblais sur sols compressibles et sur la confrontation calculs-mesures in situ. Bulletin des Laboratories des Ponts et Chaussées 2001;232:43-58.

[11] Mestat Ph. Applications de la base de données MOMIS à la validation du calcul des ouvrages souterrains. Bulletin des Laboratoires des Ponts et Chaussées 2002;236:59-75.

[12] Negro A, de Queiroz PIB. Prediction and performance: a review of numerical analyses for tunnels. In: Kusakabe $\mathrm{O}$ et al., editors. Geotechnical aspects of underground construction in soft ground. Balkema; 2000. p. 409-18.

[13] Peck RB. Deep excavations and tunnelling in soft grounds. In: 7th International Conference on Soil Mechanics and Foundation Engineering. Mexico City, State of the art report; 1969. p. $225-90$. 